
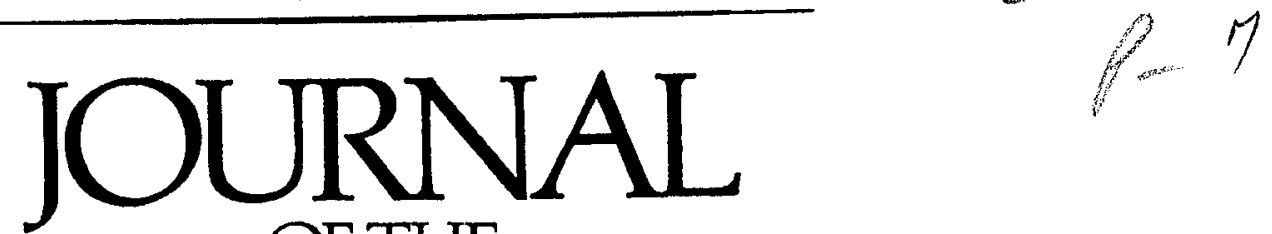

OF THE
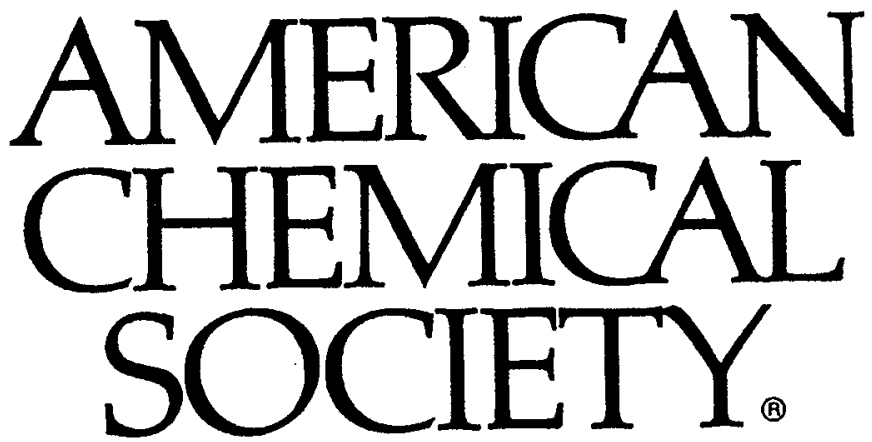

Photodeposition of Amorphous Polydiacetylene Films from Monomer Solutions onto Transparent Substrates

M. S. Paley, D. O. Frazier, H. Abdeldeyem, S. Armstrong, and S. P. McManus

Contribution from the Space Science Laboratory, NASA Marshall Space Flight Center, Huntsville, Alabama 35812 , and Materials Science Program, University of Alabama in Huntsville, Huntsville, Alabama 35899 



\title{
Photodeposition of Amorphous Polydiacetylene Films from Monomer Solutions onto Transparent Substrates
}

\author{
M. S. Paley, ${ }^{* \dagger}$ D. O. Frazier,${ }^{\dagger}$ H. Abdeldeyem, ${ }^{\dagger}$ S. Armstrong,${ }^{\dagger}$ and \\ S. P. McManus ${ }^{\ddagger}$
}

Contribution from the Space Science Laboratory, NASA Marshall Space Flight Center, Huntsville, Alabama 35812, and Materials Science Program, University of Alabama in Huntsville, Huntsville, Alabama 35899

Received October 11, 1994 ${ }^{\otimes}$

\begin{abstract}
Polydiacetylenes are a very promising class of polymers for both photonic and electronic applications because of their highly conjugated structures. For these applications, high-quality thin polydiacetylene films are required. We have discovered a novel technique for obtaining such films of a polydiacetylene derivative of 2-methyl4-nitroaniline using photodeposition from monomer solutions onto UV transparent substrates. This heretofore unreported process yields amorphous polydiacetylene films with thicknesses on the order of $1 \mu \mathrm{m}$ that have optical quality superior to that of films grown by standard crystal growth techniques. Furthermore, these films exhibit good third-order nonlinear optical susceptibilities; degenerate four-wave mixing experiments give $\chi^{(3)}$ values on the order of $10^{-8}-10^{-7} \mathrm{esu}$. We have conducted masking experiments which demonstrate that photodeposition occurs only where the substrate is directly irradiated, clearly indicating that the reaction occurs at the surface. Additionally, we have also been able to carry out photodeposition using lasers to form thin polymer circuits. In this work, we discuss the photodeposition of polydiacetylene thin films from solution, perform chemical characterization of these films, investigate the role of the substrate, speculate on the mechanism of the reaction, and make a preliminary determination of the third-order optical nonlinearity of the films. This simple, straightforward technique may ultimately make feasible the production of polydiacetylene thin films for technological applications.
\end{abstract}

\section{Introduction}

Polydiacetylenes are a class of highly conjugated organic polymers that are of considerable interest because of their unique chemical, optical, and electronic properties. ${ }^{1-3}$ They have been studied extensively as organic conductors and semiconductors, as well as nonlinear optical materials. Many of these applications require the formation of high-quality thin polydiacetylene films, i.e., films possessing minimal defects such as impurities, inhomogeneities, light scattering centers, etc. The standard techniques for obtaining polydiacetylene thin films involve the growth of crystalline diacetylene monomer films or the deposition of Langmuir-Blodgett (LB) films, followed by topochemical polymerization of these films in the solid state to yield ordered polydiacetylene films. This ability to undergo solidstate polymerization is a very intriguing property of diacetylenes; in principle, one can start with a single crystal monomer and obtain a single crystal polymer. ${ }^{4}$ However, the process is not trivial; the formation of high-quality crystalline diacetylene monomer films or LB films can be very tedious and difficult, and furthermore, by no means do all monomers polymerize readily in the solid state. ${ }^{5}$ On the other hand, techniques for obtaining amorphous films, such as spin-coating, are limited

${ }^{\dagger}$ NASA Marshall Space Flight Center.

‡ University of Alabama in Huntsville.

$\otimes$ Abstract published in Advance ACS Abstracts, April 15, 1995

(1) Polydiacetylenes; Bloor, D., Chance, R. R., Eds.; Martinus Nijhoff Publishers: Dordrecht, The Netherlands, 1985.

(2) Nonlinear Optical Properties of Organic Molecules and Crystals; Chemla, D. S., Zyss, J., Eds; Academic Press: Orlando, FL, 1987; Vol. 2. (3) Introduction to Nonlinear Optical Effects in Molecules and Polymers; Prasad, P. N., Williams, D. J., Eds.; John Wiley and Sons, Inc.: New York, 1991.

(4) Wegner, G. Z. Naturforsch. 1969, 246, 824. See also: Solid State Polymerization; Sandman, D. J., Ed.; American Chemical Society: Washington, DC, 1987.

(5) Thakur, M.; Meyler, S. Macromolecules 1985, 18, 2341. because of the insolubility of many polydiacetylenes. ${ }^{6}$ Unfortunately, no method exists which is generally applicable to the formation of polydiacetylene thin films possessing sufficiently high quality for technological applications, i.e., electronic and photonic devices.

During the course of crystal growth studies in anticipation of a space experiment, we have discovered a novel process for the formation of thin amorphous polydiacetylene films using photodeposition from monomer solutions onto transparent substrates. ${ }^{7}$ Specifically, we have directly synthesized a polymeric film from a diacetylene monomer (DAMNA) derived

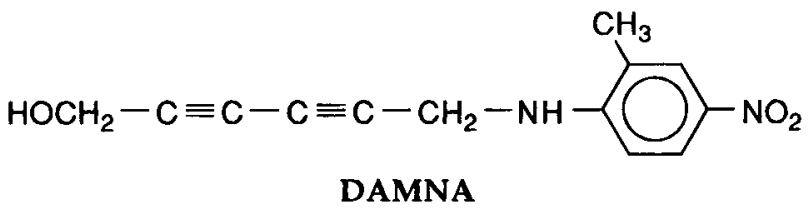

from 2-methyl-4-nitroaniline (MNA) that only sluggishly polymerizes when the crystalline monomer is irradiated. ${ }^{8}$ This compound was one of several asymmetric diacetylenes that were first studied extensively for their optical and electronic properties by Garito and co-workers in the late 1970s; however, their investigations did not include behavior in solution. ${ }^{9,10}$ We have found that thin polydiacetylene (PDAMNA) films can be obtained readily from solutions of DAMNA in 1,2-dichloro-

(6) Reference 1, p 240

(7) Paley, M. S.; Frazier, D. O.; Abdeldeyem, H.; McManus, S. P. Chem. Mater. 1994, 6 (12), 2213.

(8) Paley, M. S.; Frazier, D. O.; McManus, S. P.; Zutaut, S. E.; Sangahadasa, M. Chem. Mater. 1993, 5 (11), 1641.

(9) Horner, C. J.; Garito, A. F. Makromol. Chem. 1981, I82, 19

(10) Garito, A. F.; Singer, K. D.; Teng, C. C. In Nonlinear Optical Properties of Organic and Polymeric Materials; Williams, D. J., Ed.; ACS Symposium Series, Vol. 233; American Chemical Society: Washington, DC, 1983; pp 1-26. 


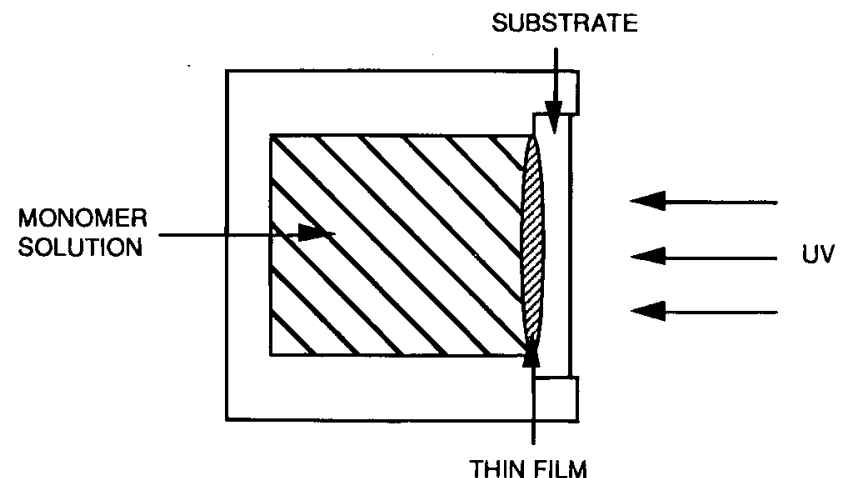

Figure 1. Diagram of the apparatus used for the photodeposition of polydiacetylene thin films from solution.

ethane by irradiation with long-wavelength UV light through a quartz or glass window, which serves as the substrate. This simple straightforward process yields transparent films with thicknesses on the order of $1 \mu \mathrm{m}$.

Despite the considerable volume of literature available on diacetylenes and polydiacetylenes, this solution-state photodeposition reaction has never been reported. Indeed, there is little mention at all of diacetylene polymerization in solution; most research has focused on solid-state polymerization because of its novelty and because of the insolubility of most polydiacetylenes. Thus there is a great deal of fundamental science to be learned about this intriguing solution photodeposition process. In the present work, we study the photodeposition of polydiacetylene (primarily PDAMNA) thin films from solution onto a variety of substrates, perform chemical characterizations of these films and of the polymer from the bulk solution, investigate the role of the substrate, speculate on the mechanism of the reaction, and make a preliminary determination of the third-order nonlinear optical properties of the films.

\section{Results and Discussion}

Thin Film Photodeposition. Because this solution photodeposition reaction is newly discovered, many of the parameters controlling its efficacy are not yet known. The basic idea is quite straightforward; the diacetylene monomer solution is irradiated through a UV transparent substrate, and a thin polydiacetylene film results. To find an appropriate UV wavelength for carrying out the reaction, a UV-vis spectrum of the monomer in the photodeposition solvent is obtained to determine where the monomer absorbs. The longest wavelength at which absorption occurs is best. The thickness of the film is determined by the intensity of the UV light and the duration of exposure. After a certain point the photodeposition reaction terminates because the polymer film reaches a thickness where insufficient UV radiation can penetrate through to continue the reaction. Hence a wavelength where the polymer's absorption is minimal is best for growing thicker films; generally this occurs at longer wavelengths.

DAMNA monomer exhibits strong absorption at $366 \mathrm{~nm}$ in 1,2-dichloroethane due to the MNA group; hence this is the most suitable wavelength for photodeposition (although shorter wavelengths can be used). Special chambers were constructed for carrying out the reaction and obtaining thin films on small round substrate disks (Figure 1). To obtain PDAMNA thin films, a solution of DAMNA in 1,2-dichloroethane is placed inside the chamber. Typically, using a hand-held $15 \mathrm{~W}$ longwave UV lamp (approximate radiation intensity of $800 \mu \mathrm{W} /$ $\mathrm{cm}^{2}$ at 6 in.) placed directly in front of the substrate, a film of approximately $0.6 \mu \mathrm{m}$ maximum thickness is obtained after 24 $\mathrm{h}$ of exposure. Thicker films can be grown if more intense UV light is used. After the photodeposition is complete, the monomer solution, which now also contains suspended particles of precipitated polymer, is removed from the growth chamber. The substrate, now coated on one side with the PDAMNA film, is then removed, washed with 1,2-dichloroethane, and dried.

Thin PDAMNA films obtained in this manner are transparent, glassy yellow-orange in appearance, suggesting an amorphous nature. Both refractive index measurements and electron beam diffraction studies indeed indicate that the films are amorphous. The films are insoluble in organic solvents, although solvents such as acetone can cause them to peel off of the substrate. Even concentrated sulfuric acid does not dissolve the films; they turn brown, shrivel, and peel off of the substrate but do not dissolve, even after several weeks. In contrast, the PDAMNA powder precipitated from the bulk solution is soluble in solvents such as acetone and DMSO.

Characterization of PDAMNA Films. Because of the insolubility of the PDAMNA films, many of the conventional methods of polymer analysis are not applicable. To aid in discerning the structure, we have obtained ${ }^{13} \mathrm{C}$-NMR spectra of the polymer from the bulk solution and have obtained UV-vis and FTIR spectra of the films.

The ${ }^{13} \mathrm{C}-\mathrm{NMR}$ spectrum of PDAMNA precipitated from the bulk solution and dissolved in DMSO- $d_{6}$ shows absorptions at 128 and $59 \mathrm{ppm}$, consistent with the presence of olefinic and acetylenic carbons, respectively. ${ }^{11}$ The monomer (also in DMSO- $d_{6}$ ) shows absorptions at $79,76,68$, and $66 \mathrm{ppm}$, due to the diacetylene backbone, but no absorption at $128 \mathrm{ppm}$.

UV-visible absorption spectra of PDAMNA films photodeposited onto quartz indicate that the films are essentially transparent at wavelengths greater than $700 \mathrm{~nm}$, then exhibit increasing absorption at shorter wavelengths, with a shoulder peak at $370 \mathrm{~nm}$ due to the MNA group, and a maximum between 200 and $300 \mathrm{~nm}$. DAMNA monomer, in 1,2-dichloroethane, is transparent above $450 \mathrm{~nm}$. Thus the red shift in the absorption band is indicative of the polymerization that has occurred. The UV-visible spectrum of PDAMNA films obtained from solution matches very well with that of PDAMNA films obtained by solid-state polymerization of polycrystalline DAMNA monomer films.

One important thing we desired to determine is the structure of the polymer. It is well-known that, in the solid state, diacetylenes polymerize by a 1,4 mechanism, giving a linear polymer with a backbone consisting of alternating doublesingle-triple bonds. ${ }^{12}$ However, in solution, there exists the additional possibility of 1,2 polymerization, giving a polymer with a backbone consisting of alternating double-single bonds (essentially a polyacetylene with acetylene side groups). It may even be the case that both types of polymerization are occurring simultaneously, yielding a polymer which has both polyacetylene and polydiacetylene character. Also, in solution, there is a greater probability of forming branched polymers.

It has been observed generally that the vibrational nature of polydiacetylenes shows no infrared active backbone absorptions and that diacetylene monomers are virtually indistinguishable from their polymers. ${ }^{13}$ This is indeed the case for DAMNA polymerized in the solid state. A sample of the polycrystalline monomer powder on $\mathrm{ZnSe}$ showed little change in its infrared spectrum upon polymerization by irradiation at $366 \mathrm{~nm}$, and nowhere in either spectrum is there a peak corresponding to a carbon-carbon triple bond (which should appear between 2000 and $2100 \mathrm{~cm}^{-1}$ ).

(11) Schaefer, J.; Sefcik, M. D.; Stejskal, E. O.; McKay, R. A.; Hall, P. L. Macromolecules 1981, 14, 557 .

(12) Reference 4.

(13) Reference 2, Vol. 2, Chapter III-3. 
A

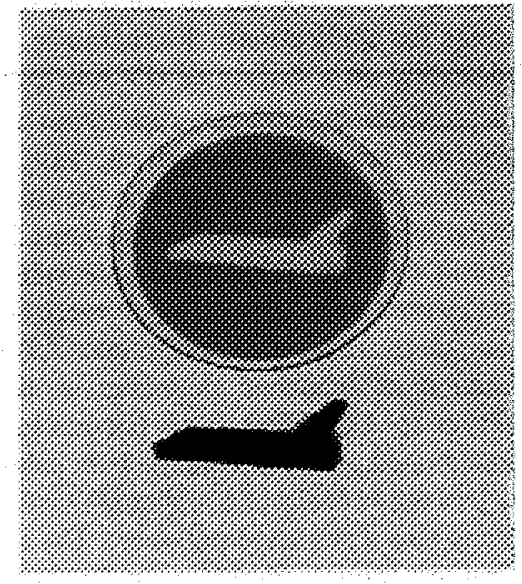

B

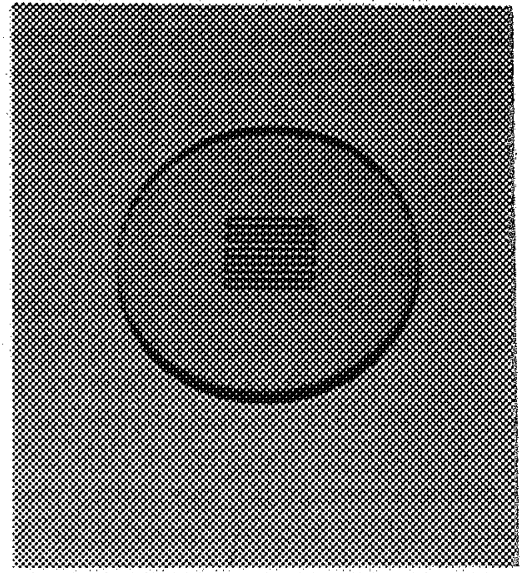

Figure 2. (A) PDAMNA film formed by photodeposition from solution onto a thin glass substrate. A portion of the substrate was blocked from exposure to the UV light by the mask (shown below the film), which was placed on the opposite side of the glass disk as the film, clearly demonstrating that photodeposition occurs only where the substrate is irradiated directly. (B) PDAMNA circuit photodeposited onto quartz using the UV radiation from an argon ion laser, a technique which could provide a novel means for producing optical circuits.

In the case of PDAMNA photodeposited from solution (onto $\mathrm{KBr}$ ), the FTIR spectrum is, not surprisingly, somewhat different from that of material polymerized in the solid state. However, there are still no absorptions present between 2000 and 2100 $\mathrm{cm}^{-1}$. Although backbone vibrations of polydiacetylenes are not typically infrared active because of symmetry considerations, side groups do generally show up. ${ }^{13}$ Thus, for 1,4 polymerization, because the only carbon-carbon triple bonds present are in the polymer backbone, they would not be expected to absorb in the IR. On the other hand, for 1,2 polymerization, the carbon-carbon triple bonds are in the side groups and thus should be expected to absorb. The absence of carbon-carbon triple bond absorptions in the FTIR spectrum of PDAMNA photodeposited from solution seems to suggest that primarily 1,4 polymerization has occurred. At this point, however, we cannot absolutely rule out that some 1,2 polymerization may also have occurred.

Role of the Substrate. The exact role that the substrate plays in photodeposition of polydiacetylene films from solution is not yet fully understood. Apparently, any substrate which is sufficiently transparent to UV light can be used; thus far we have grown PDAMNA films onto glass, quartz, mica, indium tin oxide coated glass, polyethylene teraphthalate, $\mathrm{KBr}$, and $\mathrm{NaCl}$. In order to gain some insight into the process that occurs at the surface of the substrate during photodeposition, we have conducted masking experiments in which a portion of the substrate is blocked from exposure to the UV light during film deposition. The mask is placed on the exterior surface of the substrate (opposite to the side on which the film is grown) and thus is not in contact with the solution; it serves merely to protect part of the substrate from the light. Interestingly, the result is that film deposition occurs only where the substrate is directly exposed to the light (Figure 2A); absolutely no film deposition occurs behind the mask, even though polymerization takes place throughout the bulk solution. This clearly indicates that polymerization occurs at (or very near) the surface. If this were simply a case of bulk solution polymerization, followed by adsorption of the polymer onto the substrate, the mask, because it is on the outside, should have no effect; film deposition would be expected to occur over the entire substrate.

On the basis of the above results, we thought it would be very interesting to try photodeposition using a laser. Thus the thin film growth chamber was mounted onto a computer- controlled translation stage, which had been programmed to trace out a desired pattern. The UV radiation (364 nm) from an argon-ion laser was then focused onto the substrate as the translator traced out the test pattern, resulting in a thin PDAMNA image of the pattern (Figure 2B). The thickness of the image is determined by the intensity of the laser, the translation rate, and the number of times that the pattern is traced, and the width of the lines is determined by the focal width of the laser beam.

Other Compounds. We have been carrying out experiments with a variety of diacetylene monomers and have found that their ability to form polymeric thin films in solution is highly dependent on the particular compound. Some monomers do not polymerize readily in solution, others polymerize to give a soluble polymer, and some polymerize to give precipitated polymer but no film. The reaction is also very solventdependent; for example, in 1,2-dichloroethane, transparent PDAMNA films form readily, in 2-butanone, translucent films form slowly, and in ethanol, virtually no film formation occurs (the polymer remains in solution). This demonstrates that polydiacetylene thin film photodeposition from solution depends greatly on the particular monomer/solvent combination.

One of the first compounds tested, after PDAMNA, was poly(2,4-hexadiyne-1,6-ditosylate), otherwise known as PTS. It was found that the monomer photopolymerized (at $254 \mathrm{~nm}$ ) very slowly in 1,2-dichloroethane and that what little polymer formed simply precipitated. Other solvents showed similar results; polymerization occurred slowly, and very little film deposition took place. In the case of poly(2,4-hexadiyne-1,6-diol), some film deposition did take place upon irradiation (at $254 \mathrm{~nm}$ ) of the monomer in 1,2-dichloroethane/acetone; however, the films were quite thin (less than $0.5 \mu \mathrm{m}$ ). On the other hand, polydiacetylene films were obtained readily from $1-(N$-methylanilino)-6-methyl-2,4-heptadiyn-6-ol in cyclohexane/benzene upon irradiation at $254 \mathrm{~nm}$. Lastly, polydiacetylene films were also formed readily from the $N$-methyl analog of DAMNA, 1-( $N$-methyl-4-nitroanilino)-2,4-hexadiyn-6-ol (again, in 1,2dichloroethane at $366 \mathrm{~nm}$ ).

Mechanism of Photodeposition. We are currently in the process of designing and conducting experiments to discern the kinetics and mechanism of diacetylene photopolymerization in solution and polydiacetylene thin film deposition from solution. 
Hence, at this point, we can only speculate on the mechanism on the basis of the limited information we have at present.

It has been well-established that in the solid-state diacetylenes photopolymerize by a triplet mechanism initiated via a biradical dimer. ${ }^{14,15}$ It seems reasonable that a similar mechanism also takes place in solution. Thus, UV light absorbed by a monomer molecule gives the excited singlet state, which undergoes intersystem crossing to the triplet state, and then reacts with a second monomer molecule to form the biradical dimer. This dimer then adds additional monomers to form the polymer chain, which eventually terminates, often by intramolecular rearrangement. Qualitative kinetics experiments indicate that the rate of photopolymerization is increased by the addition of triplet sensitizers such as anthracene to the monomer solution. This supports the notion of a triplet mechanism. In contrast, polymerization does not occur thermally, even in the presence of free radical initiators such as benzoyl peroxide or AIBN, making a single free radical (doublet) mechanism seem unlikely.

Nonlinear Optical Properties. On the basis of optical microscopy and refractive index measurements using wave guide mode analysis, PDAMNA thin films obtained via photodeposition from solution have good optical quality, superior to that of films grown using conventional crystal growth techniques. Considering the simplicity of photodeposition, this technique could make the production of polydiacetylene thin films for applications such as nonlinear optical devices technologically feasible. Hence we decided to investigate the nonlinear optical properties of the PDAMNA films, specifically, their third-order nonlinear optical susceptibilities.

Degenerate four-wave mixing experiments carried out at 532 $\mathrm{nm}$ on PDAMNA films obtained by photodeposition from solution (thicknesses around $1.0 \mu \mathrm{m}$ ) yield $\chi^{(3)}$ values on the order of $10^{-8}-10^{-7}$ esu. Qualitative measurements indicate that the response time is on the order of picoseconds, which is consistent with an electronic mechanism for the nonlinearity. It should be pointed out that, because $532 \mathrm{~nm}$ is in the absorption edge of the polymer, the $\chi^{(3)}$ values obtained are resonance enhanced. Typically, $\chi^{(3)}$ values for polydiacetylenes can vary by several orders of magnitude depending on the degree of resonance enhancement and other factors. ${ }^{16,17}$ The largest reported nonresonant (purely electronic) $\chi^{(3)}$ value for a polydiacetylene is on the order of $10^{-9}-10^{-10}$ esu for PTS single crystals. ${ }^{18}$ In order to obtain a valid measure of the inherent nonlinearity of the PDAMNA films, experiments need to be conducted at longer wavelengths where the polymer does not absorb (in the case of PDAMNA, above $700 \mathrm{~nm}$ ). To ascertain the true potential for device applications (the figures of merit), thorough measurements of light scattering, linear absorption, two and three-photon absorption, damage thresholds, etc., must be carried out. ${ }^{19}$ Such experiments are underway and will be the subject of future publications.

\section{Conclusions}

On the basis of the results obtained here, it is clear that photodeposition of polydiacetylene thin films from solution is a novel and interesting reaction. It is simple to carry out,

(14) Reference 1, pp 25-40.

(15) Sixl, H. Adv. Polym. Sci. 1984, 63, 49.

(16) Reference 3, pp 232-233.

(17) Etemad, S.; Baker, G. L.; Soos, Z. G. In Molecular Nonlinear Optics; Zyss, J., Ed., Academic Press: San Diego, CA, 1993.

(18) Thakur, M.; Krol, D. M. Appl. Phys. Lett. 1990, 56 (13), 1213. See also: Reference 2, Vol. 2, Chapter III-3.

(19) Stegeman, G. I.; Torruellas, W. In Electrical, Optical, and Magnetic Properties of Organic Solid State Materials; Garito, A. F., Jen, A. K.-Y., Lee, C. Y.-C., Dalton, L., Eds.; MRS Symposium Proceedings; Materials Research Society: Pittsburg, PA, 1994; pp 397-412. requires no special surface pretreatment of the substrate, and yields amorphous polymer films that possess good optical quality and have thicknesses on the order of $1 \mu \mathrm{m}$, which is a useful range for applications. Furthermore, these films can be obtained readily on a variety of UV transparent substrates.

Characterization experiments carried out on PDAMNA films photodeposited from solution indicate that predominantly 1,4 polymerization has occurred, just as in the solid-state. However, as stated earlier, it is still possible that some 1,2 polymerization may occur in solution. In the solid state, steric constraints from the crystal lattice only allow 1,4 polymerization to take place; in solution, there are no such constraints. Likewise, in solution, the formation of branched polymers is also possible. The insolubility of the PDAMNA films, contrasted with the solubility of the precipitated polymer from the bulk solution, suggests that the films probably consist of a higher molecular weight polymer, and perhaps also a branched polymer. The PDAMNA precipitated from the bulk solution most likely consists of small oligomers, probably dimers and trimers.

On the basis of this result, and especially, the masking experiments, it appears that photodeposition of polydiacetylene films from solution is a surface reaction. Most likely, excited monomer molecules, or possibly biradical dimers, become adsorbed onto the substrate and thereby initiate polymerization at the surface. The success of the masking experiment demonstrates the potential of photodeposition as a means of producing films in desired patterns. The result of the laserdeposition experiment is very exciting; eventually, this technique could provide a simple means for producing polymer circuits. At present, however, the optical quality of these circuits is not sufficiently high for practical applications; they appear quite inhomogeneous when viewed under a microscope.

On the other hand, the good optical quality of the PDAMNA films obtained by photodeposition from solution, as well as their large $\chi^{(3)}$ values, makes them good candidates for applications as NLO materials. Because the films are capable of wave guiding, they could have potential for use in integrated optics. Thus far, these films are incapable of exhibiting second-order optical nonlinearity because they are amorphous. However, it may be possible to induce orientation into the films by means such as electric field poling, surface modification of the substrate, or even modifying the polymer structure (e.g., attaching a liquid crystal moiety). Ordered polydiacetylene films not only would be capable of second-order nonlinearity but also should exhibit increased third-order nonlinearity. Additionally, electronic applications such as one-dimensional conductors require films with aligned polymer chains.

In conclusion, there is still much to be learned about photodeposition of polydiacetylene films from solution and diacetylene polymerization in solution. This report has focused primarily on PDAMNA; however, we have seen that other monomers can undergo this reaction. The structure/property relationships, the kinetics and mechanism of the reaction, the specific interaction between the surface of the substrate and the film, and the linear and nonlinear optical properties all warrant detailed investigations. We are currently conducting such investigations, and the results will be discussed in forthcoming publications.

\section{Experimental Section}

Unless otherwise stated, all reagents were purchased from Aldrich and used without further purification. The synthesis and characterization of DAMNA has been described previously. ${ }^{8,9}$ The 1,2-dichloroethane used was HPLC grade, and monomer solutions were filtered before use. All substrates (except $\mathrm{KBr}$ and $\mathrm{NaCl}$ ) were washed with 
acetone before use. Film thickness measurements were carried out using a Tencor $\alpha$-step 200 profilometer.

Photodeposition of PDAMNA Thin Films. As stated earlier, special Teflon chambers (Figure 1) were constructed for carrying out polydiacetylene thin film photodeposition from solution. The chambers used here were cylindrical in shape and closed at one end, with approximate dimensions of $0.5 \mathrm{~cm}$ depth and $1.2 \mathrm{~cm}$ i.d.; this holds between 1.0 and $1.5 \mathrm{~mL}$ of solution (of course, the size and shape are not critical). The substrate disks were circular, ranging from 1.3 to $1.7 \mathrm{~cm}$ in diameter, with thicknesses between 1 and $2 \mathrm{~mm}$. An O-ring was used to form a seal between the substrate window and the solution inside the chamber. Because many organic solvents, including 1,2dichloroethane, are permeable to common O-ring materials (such as Viton), Kalrez (a fluoroelastomer) O-rings were used for this purpose. A screw cap with another O-ring (any type can be used here) placed on the outside of the substrate was used to seal the chamber shut. The chambers were filled and emptied using a syringe through a small threaded fill hole at the back, which was sealed shut with a screw.

To grow thin PDAMNA films onto quartz disks (or any transparent substrate), the chambers were filled with a solution of DAMNA in 1,2dichloroethane $(3-4 \mathrm{mg} / \mathrm{mL})$, making sure to avoid air bubbles, and sealed shut. The chambers were then placed directly in front of a 15 W, $366 \mathrm{~nm}$ UV light (Blak-Ray model UVL-56), with the substrate window facing directly into the light. After $24 \mathrm{~h}$ of exposure, the photodeposition was complete; the chambers were then emptied, and the substrate disks, now coated on one side with the polymer film, were removed, immediately washed with dichloroethane, and allowed to dry. This procedure yielded transparent yellow-orange PDAMNA films with thicknesses of about $0.6 \mu \mathrm{m}$, measured near the center (the films tend to be thicker at the center than near the edges). Thinner films can be grown by shortening the duration of exposure to the UV light, and thicker films (around $1.0-1.5 \mu \mathrm{m}$ ) can be grown by exposure to a more intense UV source (such as Blak-Ray model B-100A). To grow the thicker films, it is best to first expose the substrate to the less intense UV light source for about 1 day and then switch to the more intense source for an additional day. This procedure yields films with better quality. PDAMNA films can also be grown using $254 \mathrm{~nm}$ radiation; however, the films do not grow as thick because the polymer absorbs more strongly at $254 \mathrm{~nm}$.

Characterization. UV-visible absorption spectra of the PDAMNA films on quartz substrates were obtained using an HP8452 photodiode array spectrometer, with blank quartz disks as the reference. A spectrum of PDAMNA was also obtained on a $\mathrm{KBr}$ disk; it appeared essentially identical to that on quartz. The spectrum of DAMNA monomer was obtained in 1,2-dichloroethane. Infrared spectra of the PDAMNA films on $\mathrm{KBr}$ substrates were obtained using a Perkin-Elmer 1600 Series FTIR. This was also used to monitor a sample of DAMNA monomer powder that was photopolymerized on $\mathrm{ZnSe}$. A ${ }^{13} \mathrm{C}-\mathrm{NMR}$ spectrum was obtained in DMSO- $d_{6}$ on a sample of PDAMNA precipitated from the bulk solution using an IBM-Bruker $200 \mathrm{MHz}$ Fourier transform spectrometer. The spent reaction mixtures from the thin film deposition chambers were combined and centrifuged, and the precipitated PDAMNA was washed with 1,2-dichloroethane and centrifuged again. The sample was then dried and dissolved in DMSO$d_{6}$ to obtain the spectrum. A spectrum was also obtained on DAMNA monomer dissolved in DMSO- $d_{6}$. In both cases the spectra were obtained after $4000-5000$ scans using a relaxation delay of $3 \mathrm{~s}$.

Other Compounds. To grow thin films of other polydiacetylenes, the same method described above for growing PDAMNA films was used. A solution of the monomer (again, $3-4 \mathrm{mg} / \mathrm{mL}$ ) in the appropriate solvent was placed inside the chamber, which was then irradiated at a suitable wavelength (determined from the UV-visible spectrum of the monomer). The specific conditions and results for various compounds were discussed earlier.

Masking Experiments. To gain a better understanding into the role of the surface during thin film photodeposition, a simple masking experiment was conducted. The chamber was filled with DAMNA monomer solution as described previously, only this time a small stirring bar was also placed in the chamber. A very thin glass disk (cut from a microscope slide cover slip) was used as the substrate in order to minimize the effects of refraction of the UV light around the edges of the mask; the thinner the substrate disk, the sharper the image. A small cutout from a sheet of aluminum was used as the mask, which was held in place on the exterior surface of the glass substrate disk using a quartz disk (i.e., the mask was "sandwiched" in place between the glass disk and the quartz disk). The chamber was then irradiated with the UV light while the monomer solution inside was stirred to ensure thorough mixing. After $24 \mathrm{~h}$, the reaction was stopped, the disks and the mask were removed, and the glass substrate disk was washed and dried as before. The result was that polydiacetylene film deposition took place only where the substrate was directly exposed to the UV light; there was no film deposition behind the mask (Figure 2A). Polymerization still took place throughout the bulk solution.

Laser Photodeposition. The chamber was filled with DAMNA monomer solution, using a quartz disk as the substrate, and then mounted onto a Lintech two-axis translation stage. Utilizing a $50 \mathrm{~mm}$ focal length lens, the beam from a Spectra-Physics beamlok 2060 argonion laser tuned to $364 \mathrm{~nm}$ was focused to a pinpoint on the quartz disk. The command-control system consisted of a Compumotor AT6400 board and indexer, and Compumotor $S$ drives and encoders for each axis of motion. The board was installed in an IBM 386 computer and communicated with the translation stage via the indexer. The resolution of the translation stage is $0.2 \mu \mathrm{m} / \mathrm{step}$, and the 6000 Series software provided with the Compumotor indexer enabled various motion-control programs to be utilized. By varying the intensity of the laser, the speed of the translation stage, and the number of repetitions, PDAMNA patterns with different thicknesses could be obtained. The width of the lines was adjusted by changing the focus of the laser beam.

Kinetics Experiments. In order to gain some insight into the mechanism of diacetylene photopolymerization in solution and polydiacetylene thin film photodeposition from solution, some simple qualitative kinetics experiments using triplet sensitizers and free radical initiators were performed. Two identical chambers were prepared as before, using quartz disks as substrates. Each chamber contained DAMNA monomer solution $(3.5 \mathrm{mg} / \mathrm{mL})$; however, one also contained a trace amount (at $10^{-4} \mathrm{M}$ ) of anthracene, a triplet sensitizer. The two chambers were placed side by side and irradiated for $2 \mathrm{~h}$ at $254 \mathrm{~nm}$ (this wavelength was chosen to ensure that the anthracene would absorb). The reaction was then stopped, the solutions and quartz disks (with films) were removed, and the disks were washed and dried. It was apparent from visual inspection that the solution containing anthracene had polymerized to a greater extent than that without anthracene. Furthermore, the PDAMNA film grown in the presence of anthracene was thicker (by about $30 \%$, based on UV-visible absorption spectra) than that grown under identical conditions without anthracene. Hence the addition of anthracene does cause an increase in the rate of reaction, which suggests that a triplet mechanism is operative in solution.

The experiment was repeated, only this time benzoyl peroxide was used in one of the solutions. Again, by visual inspection, it was apparent that the solution containing benzoyl peroxide had polymerized to a greater extent (even more so than in the case of anthracene). Likewise, the PDAMNA film grown from the solution containing benzoyl peroxide was about twice as thick as that grown from the control solution. Thus benzoyl peroxide causes an even greater increase in the rate of the reaction than does anthracene. Because benzoyl peroxide can behave photochemically as both a triplet sensitizer and a free radical initiator, it was decided to see if polymerization could be carried out thermally (in the dark) with benzoyl peroxide. Under these conditions only a free radical mechanism is possible. Thus a solution of DAMNA with benzoyl peroxide, same concentrations as before, was placed in a sealed glass container, wrapped in aluminum foil to keep out light, and heated at $70^{\circ} \mathrm{C}$. Even after $24 \mathrm{~h}$, no polymerization took place. The experiment was repeated using AIBN instead of benzoyl peroxide; again, virtually no polymerization occurred. Thus a single free radical mechanism seems very unlikely.

Nonlinear Optical Measurements. The linear refractive index of the PDAMNA films (on quartz) was determined by wave guide mode analysis using a Metrocon 2010 prism coupler, which can also give the film thicknesses. A value of $1.696(632 \mathrm{~nm})$ was obtained for the refractive index, and the sample thicknesses ranged between 0.5 and $1.5 \mu \mathrm{m}$, which is consistent with the values obtained from profilometry.

Optical phase modulation by degenerate four-wave mixing has been found to be a very useful technique for measuring third-order nonlinear 
optical susceptibilities. ${ }^{20}$ This technique was first developed by Helwarth in 1977, and the details are described elsewhere. ${ }^{21}$ The phase conjugation of the PDAMNA films was measured using the secondharmonic $(532 \mathrm{~nm})$ from a pulsed Nd-Yag laser at $10 \mathrm{~Hz}$ and $20 \mathrm{~ns}$ pulse duration, and the $\chi^{(3)}$ values were calculated by means of ${ }^{22}$

$$
\chi^{(3)}=\frac{2 n^{2} c \lambda \alpha \mathrm{e}^{\alpha L / 2}}{3 \pi \mu_{0}\left(1-\mathrm{e}^{-\alpha L}\right)} \sqrt{\frac{I_{4}}{I_{1} I_{2} I_{3}}}
$$

where $I_{1}, I_{2}, I_{3}$, and $I_{4}$ are the intensities of the two pump, probe, and

(20) Optical Phase Conjugation; Fisher, R. A., Ed.; Academic Press: San Diego, CA, 1983.

(21) Hellwarth, R. J. Opt. Soc. Am. 1977, 67, 1.

(22) Caro, R. G.; Gower, C. IEEE J. Quantum Electron. 1982, QE-18(9), 1376. phase conjugate signals, respectively, $c$ is the speed of light, $\mu_{0}$ is the magnetic permeability of vacuum, $n$ is the linear refractive index, $\lambda$ is the laser wavelength, $\alpha$ is the absorption coefficient, and $L$ is the film thickness. The absorption coefficient, $\alpha\left(1.474 \times 10^{6} \mathrm{~m}^{-1}\right)$, was determined from the UV-visible spectrum of the film using Beer's Law. The $\chi^{(3)}$ value of $\mathrm{CS}_{2}$, a known standard, was also determined, and the measured value of $2.8 \times 10^{-12}$ esu agrees well with the published value of $2.4 \times 10^{-12} \mathrm{esu}^{23}$ The $\chi^{(3)}$ values measured for the PDAMNA films have remained stable at around $10^{-8}$ esu for several months (storing the films in the dark).

Acknowledgment. The authors greatfully acknowledge the contributions of David Donovan, Curtis Banks, Bill Witherow, Bruce Hovanes, Don Henderson, and Rixiang Mu to this work.

\section{JA943329R}

(23) Optical Electronics, 3rd ed.; Yariv, A., Ed.; Holt, Rinehart, and Winston: New York, 1985. 
